Mathematical Modelling and Analysis

Volume 16 Number 2, June 2011, 233-247

Doi:10.3846/13926292.2011.579187

(C) Vilnius Gediminas Technical University, 2011
www.tandf.co.uk/journals/TMMA

Publisher: Taylor\&Francis and VGTU

Online ISSN: 1648-3510

Print ISSN: 1392-6292

\title{
Genetic Algorithm-based Calibration of Reduced Order Galerkin Models
}

\author{
Witold Stankiewicz ${ }^{a}$, Robert Roszak ${ }^{a}$ \\ and Marek Morzyński ${ }^{a}$ \\ ${ }^{a}$ Institute of Combustion Engines and Transport, \\ Poznan University of Technology \\ Piotrowo 3, 60-965 Poznań, Poland \\ E-mail(corresp.): stankiewicz@stanton.ice.put.poznan.pl
}

Received October 11, 2010; revised March 25, 2011; published online May 1, 2011

\begin{abstract}
Low-dimensional models, allowing quick prediction of fluid behaviour, are key enablers of closed-loop flow control. Reduction of the model's dimension and inconsistency of high-fidelity data set and the reduced-order formulation lead to the decrease of accuracy. The quality of Reduced-Order Models might be improved by a calibration procedure. It leads to global optimization problem which consist in minimizing objective function like the prediction error of the model.

In this paper, Reduced-Order Models of an incompressible flow around a bluff body are constructed, basing on Galerkin Projection of governing equations onto a space spanned by the most dominant eigenmodes of the Proper Orthogonal Decomposition (POD). Calibration of such models is done by adding to Galerkin System some linear and quadratic terms, which coefficients are estimated using Genetic Algorithm. Keywords: Navier-Stokes equations, dynamical system, numerical analysis.
\end{abstract}

AMS Subject Classification: 76D55.

\section{Introduction}

Numerical analysis of fluid flow, based on the Direct Numerical Simulation (DNS) of Navier-Stokes or Euler equations, Large Eddy Simulation (LES) or even Reynolds-averaged Navier-Stokes (RANS) analysis with various turbulence models, are very time-consuming - especially when complex geometries (like full aeroplane configuration) are analyzed. These high-fidelity models require computational grids of thousands (2D) or millions (3D) of degrees of freedom to cover all important vertex scales.

In the cases when an approximate flow solution is satisfactory (e.g. aeroelastic analysis [22]), or solution time is crucial (e.g. real-time, feedback flow control [15]), low-fidelity, Reduced Order Models (ROMs) might be used.

The truncation of mode basis and the inconsistency of reduced order formulation and full-dimensional data lead to a loss of quality, that might be 
improved using model calibration.

\section{Reduced Order Modelling}

\subsection{Galerkin method}

One of the most widely used methods of flow model reduction is projection of the residual of governing equation (2.1):

$$
\dot{\mathbf{u}}^{[N]}+\nabla \cdot\left(\mathbf{u}^{[N]} \otimes \mathbf{u}^{[N]}\right)+\nabla p^{[N]}-\nu \triangle \mathbf{u}^{[N]}=R^{[N]},
$$

approximated by a base flow $\mathbf{u}_{0}$ and a finite number of empirical, physical or mathematical modes $\mathbf{u}_{i}$ (describing the disturbance) (2.2):

$$
\mathbf{u}^{[N]}(t)=\mathbf{u}_{0}+\sum_{i=1}^{N} a_{i}(t) \mathbf{u}_{i}=\sum_{i=0}^{N} a_{i}(t) \mathbf{u}_{i}, \quad a_{0} \equiv 1,
$$

onto the space spanned by these selected modes, called Galerkin Projection $[15,24,33]$. In a Hilbert space, the reduction is done by the computation of inner products of residual $R^{[N]}$ and each of the modes $\mathbf{u}_{i}$, and equating them to zero $(2.3)$ :

$$
\left(\mathbf{u}_{i}, R^{[N]}\right)_{\Omega}:=\int_{\Omega} \mathbf{u}_{i} R^{[N]} d \Omega=0 .
$$

This approach leads to the system of ordinary differential equations (Galerkin System) (2.4), linking the mode (Fourier) coefficients $a$, their time derivatives $\dot{a}$ and constant parameters $l_{i j}$ and $q_{i j}$ :

$$
\dot{a}_{i}=\nu \sum_{j=0}^{N} l_{i j} a_{j}+\sum_{j=0}^{N} \sum_{k=0}^{N} q_{i j k} a_{j} a_{k},
$$

where $l_{i j}=\left(\mathbf{u}_{i}, \triangle \mathbf{u}_{j}\right)_{\Omega}, q_{i j k}=-\left(\mathbf{u}_{i}, \nabla \cdot\left(\mathbf{u}_{j} \otimes \mathbf{u}_{k}\right)\right)_{\Omega}$.

In the case of absolutely unstable wake flow, the pressure-term is neglected. A comprehensive study on low-dimensional pressure-term representation might be found in [25].

\subsection{Proper orthogonal decomposition}

The most popular mode basis used in Reduced Order Modelling of the flow results from Proper Orthogonal Decomposition [3] of snapshots (Fig. 1, topleft) captured from numerical simulation or experiment.

The method is based on the assumption of correlation between $M$ successive snapshots $\mathbf{v}_{i}$ of the flow. These flow vectors (of size $N$ ) are centered by subtracting the time-averaged solution $\overline{\mathbf{u}}$ (Fig. 1, top-right):

$$
\dot{\mathbf{v}}_{i}=\mathbf{v}_{i}-\overline{\mathbf{u}}, \quad i=1, \ldots, M .
$$

Resulting vectors $\dot{\mathbf{v}}_{i}$ describe the fluctuations in the flow and are used to compute the autocorrelation matrix $C$ of size $N \times N$ :

$$
C=\frac{1}{M} S S^{T}, \quad \text { where } S=\left[\dot{\mathbf{v}}_{1}, \dot{\mathbf{v}}_{2}, \ldots, \dot{\mathbf{v}}_{M}\right] .
$$


The solution of standard eigenproblem $C \mathbf{u}=\lambda I \mathbf{u}$ yields eigenvalues $\lambda$ and eigenvectors $\mathbf{u}$ (POD modes, Fig. 1), that form a complete, orthonormal set.
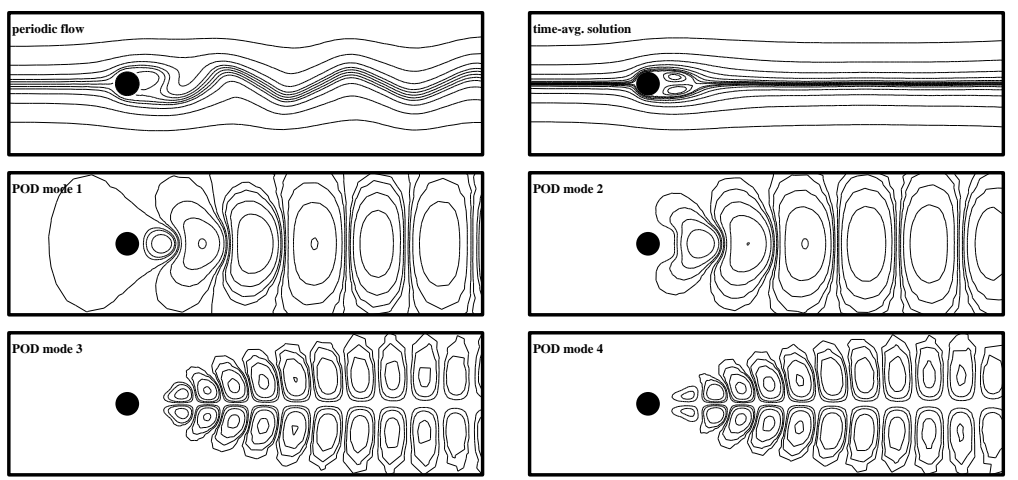

Figure 1. Streamlines of a snapshot (top-left), time-averaged solution (top-right) and first four POD modes for an incompressible flow around circular cylinder.

In the cases, where number of snapshots $M$ is mush smaller than the number of degrees of freedom $N$, snapshot technique of Sirovich [32] is used.

\section{Model Calibration}

The POD mode basis is calculated for a given set of snapshots of unsteady flow is truncated by keeping only the most energetic modes in the POD expansion, corresponding to the largest eigenvalues $\lambda$. The neglect of small (dissipative) scales results in filtering of high frequencies (e.g. turbulence) and vanishing of energy transfers between resolved and unresolved scales of fluid flow [8].

Additional differences might be caused by the structural instability of Galerkin Projection $[19,24,29]$ and the inconsistency of data set and the reducedorder formulation, resulting from the neglect or inaccurate treatment of pressure and boundary terms, or not verified incompressibility of the flow at small Mach numbers [7]. The discrepancies between the Reduced-Order Galerkin model and a high-fidelity model (like Direct Numerical Simulation of NavierStokes equations or Large Eddy Simulation) distort the frequencies, phases and amplitudes of mode coefficients, that results in different frequency of vortex shedding, under- or overestimation of turbulent kinetic energy level and different dynamical responses.

To correct the behaviour and improve the accuracy of Reduced Order Galerkin Model, the coefficients of the Galerkin system of ODE might be adjusted [8]. One of the ways of such a calibration is the use of artificial, "eddy" viscosities to recover the effects of truncated modes $[1,27,28]$. Such an artificial viscosity might be considered as a constant value $\nu_{T}$, or $N$ parameters $\nu_{T, i}$ related to each one of the modes:

$$
l_{i j}^{+}=\frac{\nu_{T}}{\nu} l_{i j} \quad \text { or } \quad l_{i j}^{+}=\frac{\nu_{T, i}}{\nu} l_{i j}, \quad i=1, \ldots N .
$$


All linear coefficients $l_{i j}^{+}$are calibrated by Galletti et al. [14] in order to model the terms relevant to the pressure in Galerkin System of laminar flow past a square cylinder. To improve the results of calibration, both linear and quadratic coefficients $q_{i j k}^{+}$of Galerkin System might be modified [8].

The resulting system of equations might be written as follows:

$$
\dot{a}_{i}=\nu \sum_{j=0}^{N}\left(l_{i j}+l_{i j}^{+}\right) a_{j}+\sum_{j=0}^{N} \sum_{k=0}^{N}\left(q_{i j k}+q_{i j k}^{+}\right) a_{j} a_{k} .
$$

In Reduced-Order Modelling, the objective function to be minimized is the prediction error of the model. It can be chosen as mean square error of the mode coefficients (3.2) or their time-derivatives (3.3):

$$
\begin{aligned}
& \chi_{0}:=\sum_{i=1}^{N} \int_{0}^{T}\left(a_{i}^{R O M}(t)-a_{i}^{D N S}(t)\right)^{2} d t=\text { Min }, \\
& \chi_{1}:=\sum_{i=1}^{N} \int_{0}^{T}\left(\dot{a}_{i}^{R O M}(t)-f_{i}\left(\mathbf{a}^{D N S}(t)\right)\right)^{2} d t=\mathrm{Min},
\end{aligned}
$$

where $a_{i}^{R O M}$ and $a_{i}^{D N S}$ represent mode coefficients for Galerkin Model and reference simulation. These definitions can be referred as Floquet and Poincaré calibration, respectively $[4,23]$. Another error definitions might based on the turbulent kinetic energy:

$$
\chi_{2}:=\int_{0}^{T}\left(\sum_{i=1}^{N}\left(a_{i}^{R O M}(t)\right)^{2}-\sum_{i=1}^{N}\left(a_{i}^{D N S}(t)\right)^{2}\right)^{2} d t=\operatorname{Min}
$$

or modal energy flow balance:

$$
\chi_{3}:=\sum_{i=1}^{N}\left(P_{i}+C_{i}+D_{i}+T_{i}+F_{i}\right)^{2}=\text { Min, }
$$

where $P_{i}, C_{i}, D_{i}, T_{i}$ and $F_{i}$ represent modal production, convection, dissipation, transfer and pressure power, respectively, leading to E-flow calibration, proposed by Bernd R. Noack [23].

\section{Global Optimization}

The goal of global optimization algorithms is to find the best possible element in search space according to a set of objective functions (criteria). These functions might be both pure mathematical expressions or algorithms involving multiple simulations [36].

The probabilistic global optimization algorithms are useful in the cases where the relation between a solution candidate and its "fitness" is complicated or the dimension of search space is very high. Many of these algorithms are inspired by the nature. Genetic Algorithms [5, 6, 13, 18, 30] and Evolutionary Programming [12] mimic the evolution of life on Earth, while swarm 
intelligence algorithms like Particle Swarm Optimization [11, 20, 31, 35] and Ant Colony Optimization [10,21] simulate the behaviour of individuals of a biological system, that spread in the environment looking for a food.

Another example of nature-based optimization is based on remodelling of trabecular bones [26], and an exhaustive survey of global optimization algorithms can be found in [36].

\section{Genetic Algorithms}

\subsection{Data encoding}

Genetic algorithm (Fig. 2) starts from an initial population, whose individuals (candidate solutions) usually are randomly generated and vary in the values of parameters (encoded in genes of chromosome), and is repeated until convergent solution is found or for a given number of generations.

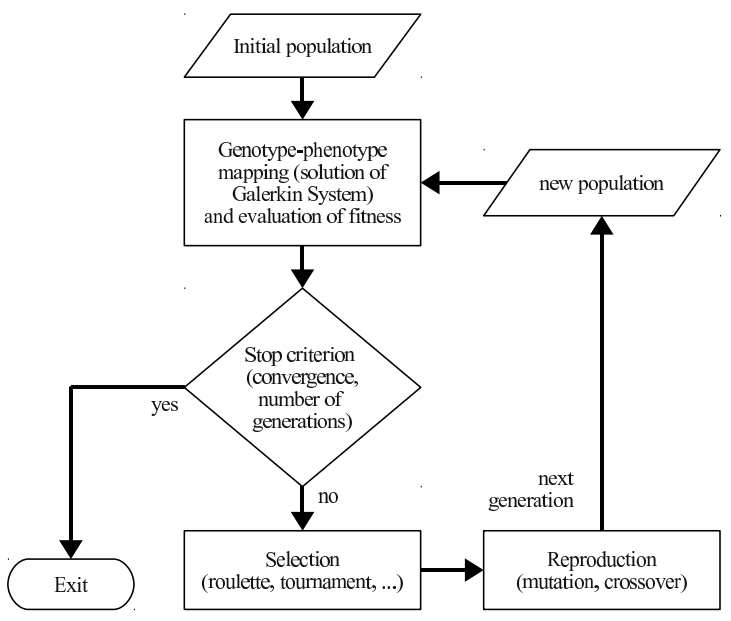

Figure 2. Flow chart of genetic algorithm.

The chromosomes of each individual, storing the information about the parameters to be changed (the search space), consists of a number of genes. They are usually encoded as binary strings (with the number of bits dependent on the required accuracy and an expected range of values in the population), but real-number another genetic representations are also possible.

In the calibration of Galerkin models presented here, the genes are encoded using double-precision real numbers. The number of genes vary from one (calibration of "eddy" viscosity $\left.\nu_{T}\right)$ to $N(N+1)(N+2)$ (calibration of linear and quadratic coefficients $l_{i j}^{+}$and $q_{i j k}^{+}$).

\subsection{Genetic operators}

In each generation a number of genetic operations, like mutation and crossover, are performed on the parent population to form an offspring. 
Mutation is used to preserve the genetic diversity of the population and to prevent the individuals from becoming too similar to each other by introducing small changes into their genotypes. This diversification allows to avoid local minima. In the case of binary encoding, values of randomly selected bits are toggled, and for real-number encoding the modification can be done by replacing (with a given probability) the value of a gene with a number drawn from a normal distribution [36].

Crossover operator allows recombination of chromosomes, that is done by swapping parts of two genotypes (Fig. 3). For binary-encoded chromosomes, single-point (SPX), two-point (TPX) and multi-point crossover (MPX) might be considered. In the first case, both chromosomes are split in the same one crossover point and the second parts of genotypes are replaced. In two-point crossover the part of chromosome between two crossover points is replaced, and multi-point crossover is generalized, $n$-point recombination of genes.
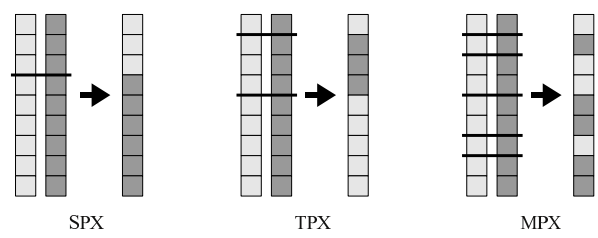

Figure 3. Sketch of crossover operation.

In real-number encoding, crossover is done by averaging the values of a certain number of genes, while keeping other genes unchanged.

\subsection{Evaluation of fitness function}

After applying genetic operators, new generation of individuals is created. For each individual of current population the genotype-phenotype mapping is performed (in order to get the observable characteristics in the problem space basing on the parameters from the search space) and the fitness (objective) function is evaluated. This step is problem-specific, and in the case of ReducedOrder Model calibration it requires the solution of a new Galerkin system and calculation of prediction error.

The selection algorithms (described in Section 5.4) assume that the largest values of fitness function are related to the best solutions. In respect of this, the fitness function used here have been chosen as an inverse of $\chi_{0}(3.2)$ in the case of Floquet calibration and $\chi_{1}(3.3)$ in the case of Poincaré calibration.

\subsection{Selection methods}

The final step is the selection of the fittest individuals to further modifications. Among many selection algorithms, whose overview might be found in $[16,36]$, the fitness proportionate reproduction, ranking, truncation and tournament are the most widely used.

Roulette wheel selection [9] is the most popular variant of fitness proportionate reproduction. The fitness function is normalized for each individual by 
dividing it by the sum of all fitness values, so that sum of fitness functions for whole population equals 1 . The share of the imaginable roulette wheel (Fig. 4) corresponding to each individual is proportional to it's normalized value of fitness function.

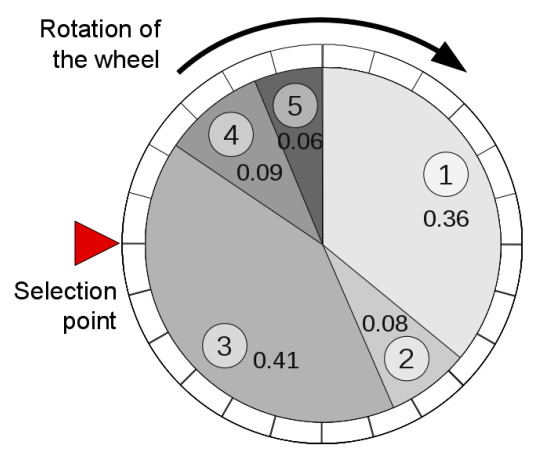

Figure 4. Example of roulette wheel selection.

Then a random real number from a range $\langle 0 \cdots 1\rangle$ is chosen, and the individual whose accumulated normalized fitness value is grater than this random number is selected for reproduction.

In the case of minimization problems, small values of error functions like $\chi_{0}$ and $\chi_{1}$ denote better solutions. The possible modifications of the method are different definition [36] of probability of individual's selection:

$$
P(i)=\frac{\max (\chi)-\chi(i)}{N_{p o p} \max (\chi)-\sum_{j=1}^{N_{p o p}} \chi(j)},
$$

or the use of inverse of error prediction $\chi$ as the fitness function, as mentioned in Section 5.3.

In ranking selection [2], the individuals are sorted according to the value of fitness function. Next, each individual is copied number of times corresponding to it's position, and proportionate reproduction according to this assignment is performed.

Truncation selection is based on the selection of a part (e.g. half, one-fifth, etc.) of the population with the fittest individuals and it's duplication as long as the size of population reaches it's previous value.

Tournament selection [17] is one of the most popular and effective selection schemes [36]. A certain number $k$ of individuals are taken from the population. From each group the best individual is selected for reproduction. The larger $k$, the more copies of the fittest individuals will be used in reproduction, and the more unfit elements will be discarded.

In this work, a combined truncation - roulette wheel selection is used. To improve convergence, elitism is taken into account by retaining the best individual of the population unchanged in the next generation. 


\section{Application of Genetic Algorithm in the Calibration of Reduced Order Models}

\subsection{Test-case description}

In this paper, Reduced Order Models of an incompressible flow around circular cylinder are calibrated using genetic algorithm described in Section 5. Reynolds number, related to the diameter of the cylinder, is $R e=100$, what means an unstable flow with limit cycle oscillations. Snapshots of that flow (calculated with the time step $d t=0.1$ ) are used to get POD modes (Fig. 1, top-right). The truncation of mode basis as well as the neglect of pressure term affect the model quality. The fitness functions are based on mean square errors of mode coefficients $a_{i}\left(\chi_{0}\right.$, Floquet calibration $)$ and their time derivatives $\partial a_{i} / \partial t\left(\chi_{1}\right.$, Poincaré calibration) for 1200 snapshots of periodic flow.

\subsection{Calibration of three-equation Galerkin model}

Two Galerkin models are used. The first one (Fig. 5) uses time-averaged solution, the two most energetic POD modes and additional shift-mode [34], that stabilises the model.
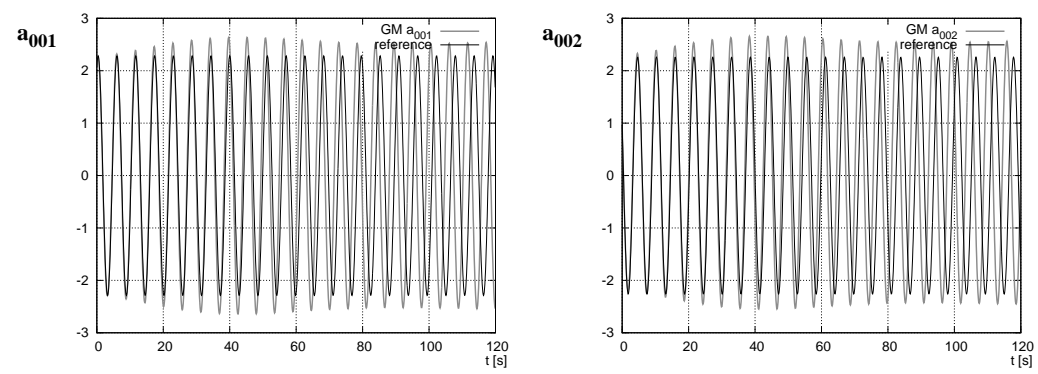

Figure 5. Uncalibrated three-equation Galerkin model: values of Galerkin model's mode coefficients compared to their reference DNS values.

In the case of the three-equation model the purpose of calibration is to minimize the model's prediction error by adjusting the frequency of vortex shedding (that is slightly smaller than reference one) and the value of Turbulent Kinetic Energy (that has to be reduced). Multiple optimizations have been done with various, experimentally chosen values of Genetic Algorithm's parameters like the size of population, probabilities of mutation and crossover and the number of modified genes. In Tab. 1 the parameters giving the best results in the calibration of "eddy" viscosity $\left(\nu_{T}\right)$, linear terms $\left(l_{i j}^{+}\right)$and both, linear and quadratic terms are gathered.

$N_{\text {Pop }}$ means the size of population, $N_{G e n}$ is the number of performed iterations of genetic algorithm, $P_{\text {crossover }}$ and $P_{\text {mutation }}$ represent the chosen probabilities of genetic operations. Number of individual's genes modified in each generation and a total number of individual's genes are given by $N_{\text {genes }}$ and $X_{\text {mutation }}$ describes the range of possible mutations of gene. 
Table 1. Parameters of three-equation model calibration.

\begin{tabular}{lcccccc}
\hline calibration & $N_{\text {Pop }}$ & $N_{\text {Gen }}$ & $P_{\text {crossover }}$ & $P_{\text {mutation }}$ & $N_{\text {genes }}$ & $X_{\text {mutation }}$ \\
\hline$\chi_{1}: \nu_{T}$ & 30 & 2 & 0.7 & 0.5 & $1 / 1$ & $500 \%$ \\
$\chi_{0}: l_{i j}^{+}$ & 70 & 5 & 0.7 & 0.35 & $5 / 12$ & $100 \%$ \\
$\chi_{0}: l_{i j}^{+}$and $q_{i j k}^{+}$ & 150 & 10 & 0.7 & 0.35 & $15 / 60$ & $100 \%$ \\
\hline
\end{tabular}

The Poincaré calibration has been used in optimization of "eddy" viscosity. The resulting model (Fig. 6), with $\nu_{T}=0.207 \nu$, has adjusted frequency and phase of coefficients for both POD modes, but the amplitude is still overestimated. The prediction errors $\chi_{0}$ and $\chi_{1}$ for this calibrated model are 200 times smaller compared to the uncalibrated model.
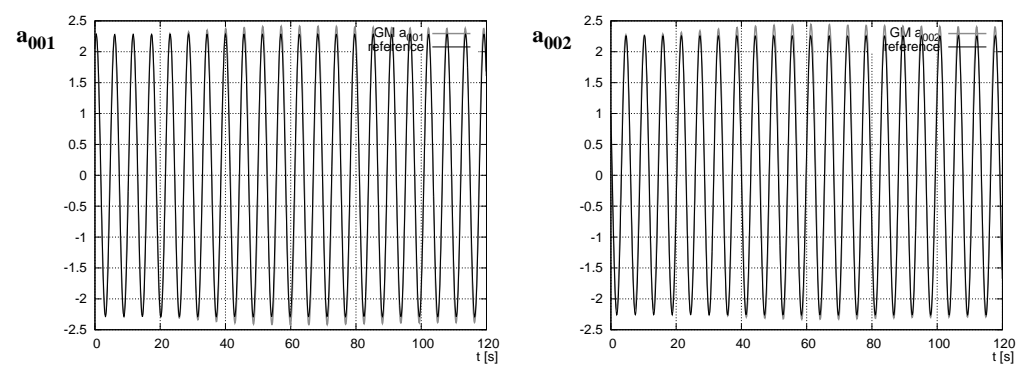

Figure 6. Three-equation Galerkin model with "eddy" viscosity calibration.

Even better results have been obtained using Floquet calibration to modify linear and quadratic terms of the Galerkin system. In that case, the convergence of genetic algorithm have been obtained in $10^{\text {th }}$ generation (Fig. 7). The

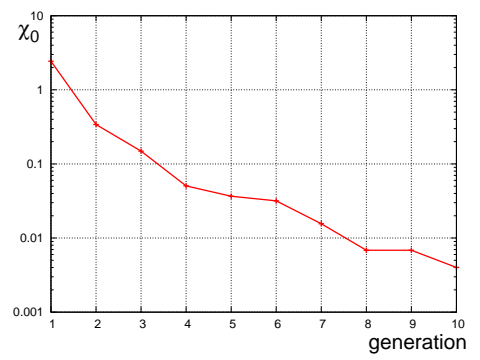

Figure 7. Prediction error $\chi_{0}$ for three-equation Galerkin model with Floquet calibration of $l_{i j}^{+}$and $q_{i j k}^{+}$terms in consecutive iterations of genetic algorithm.

resulting model (Fig. 8) has the error $\chi_{0}$ one thousand times smaller than in the case of uncalibrated one. The prediction errors for uncalibrated and calibrated Reduced Order Galerkin Models of the flow around circular cylinder are gathered in Tab. 2 . 

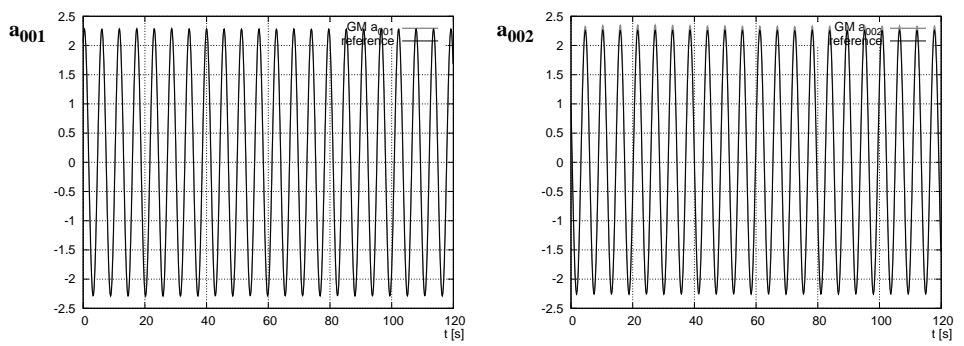

Figure 8. Three-equation Galerkin model with $l_{i j}^{+}$and $q_{i j k}^{+}$terms calibrated.

Table 2. Three-equation ROM prediction errors.

\begin{tabular}{lccc}
\hline Reduced Order Model & $\chi_{0}$ & $\chi_{1}$ & $\chi_{2}$ \\
\hline uncalibrated & 6.676285 & 8.415003 & 4.294739 \\
$\nu_{T}$ calibration & 0.034475 & 0.039484 & 0.075132 \\
$l_{i j}^{+}$calibration & 0.013826 & 0.013340 & 0.028083 \\
$l_{i j}^{+}$and $q_{i j k}^{+}$calibration & 0.004008 & 0.000883 & 0.010219 \\
\hline
\end{tabular}

\subsection{Calibration of two-equation Galerkin model}

The second Galerkin model, based on time-averaged solution and two POD modes only (two-equation model), is structurally unstable Fig. 9. The calibration is used here to stabilize the model and to minimize the prediction error.
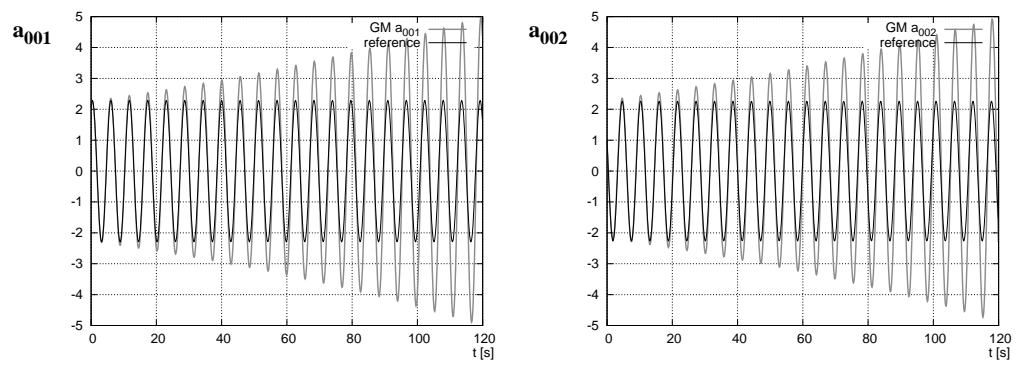

Figure 9. Uncalibrated two-equation Galerkin model compared with Direct Numerical Simulation.

The calibration of this model has been performed with a set of parameters (Tab. 3) similar to the ones shown in previous section.

Once again, (Floquet) calibration of "eddy" viscosity $\left(\nu_{T}=0.252 \nu\right.$, Fig. 10) makes the prediction errors smaller. The model is stabilized, while the frequency and phase of oscillation are kept unchanged. Poincaré calibration of both linear and quadratic terms of two-equation Galerkin System required 12 iterations (generations) of genetic algorithm (see, Fig. 11a) to get the optimized model (Fig. 12), that is almost indistinguishable from the reference data in the 
Table 3. Parameters of two-equation model calibration.

\begin{tabular}{lcccccc}
\hline calibration & $N_{\text {Pop }}$ & $N_{\text {Gen }}$ & $P_{\text {crossover }}$ & $P_{\text {mutation }}$ & $N_{\text {genes }}$ & $X_{\text {mutation }}$ \\
\hline$\chi_{0}: \nu_{T}$ & 30 & 3 & 0.7 & 0.5 & $1 / 1$ & $500 \%$ \\
$\chi_{0}: l_{i j}^{+}$ & 70 & 3 & 0.7 & 0.5 & $3 / 6$ & $75 \%$ \\
$\chi_{1}: l_{i j}^{+}, q_{i j k}^{+}$ & 150 & 12 & 0.7 & 0.35 & $12 / 24$ & $100 \%$ \\
\hline
\end{tabular}
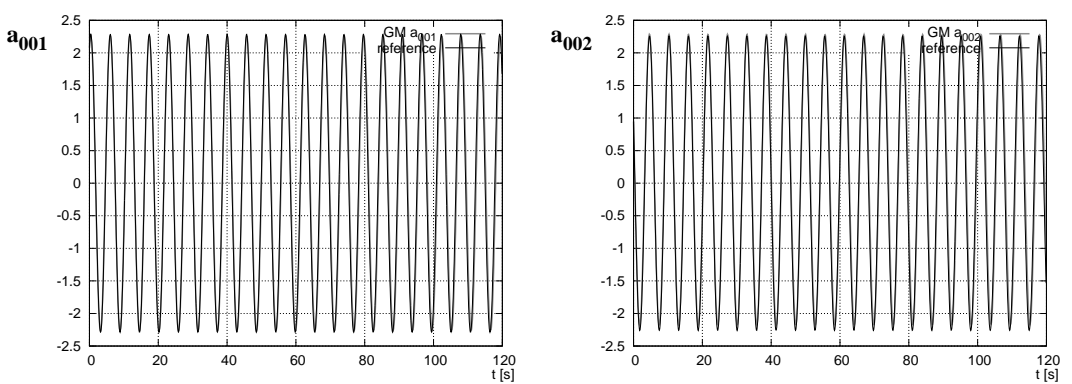

Figure 10. Two-equation Galerkin model with "eddy" viscosity calibration.

given time frame.

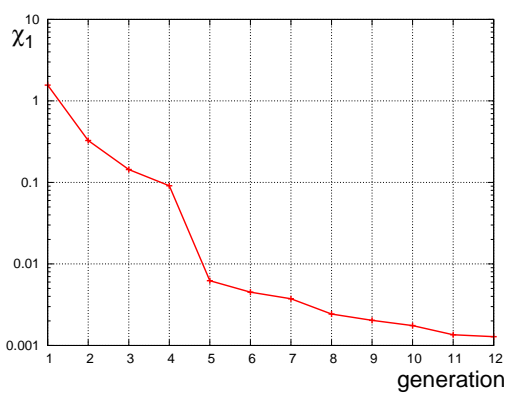

a)

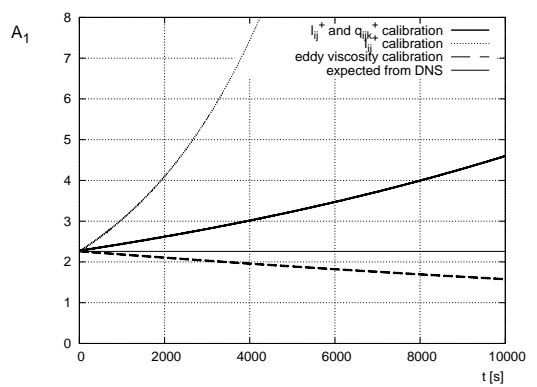

b)

Figure 11. Results of experiments: a) prediction error $\chi_{1}$ for two-equation Galerkin model with Poincaré calibration of $l_{i j}^{+}$and $q_{i j k}^{+}$terms in consecutive iterations of genetic algorithm, b) amplitude of first mode coefficient for two-equation Galerkin models.

The prediction errors for uncalibrated and calibrated two-equation Reduced Order Galerkin Models of the flow are gathered in Tab. 4.

The 2-equation Galerkin Models seem to be still unstable for longer simulation times. After one million of time steps (see, Fig. 11b) the amplitude of oscillation is almost two times larger than expected from DNS in the case of calibration of both linear and quadratic terms of Galerkin System and 1.4 times smaller in the case of "eddy" viscosity calibration. In both cases, this is still a great improvement compared to the uncalibrated 2-equation model (see, 

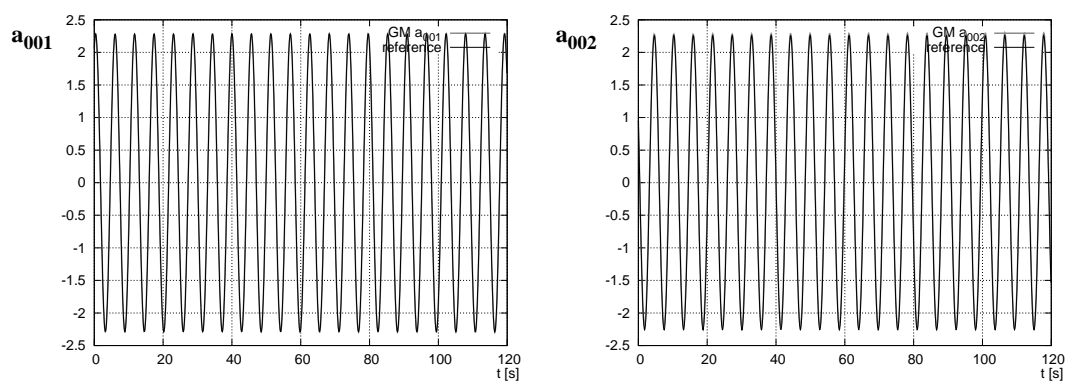

Figure 12. Two-equation Galerkin model with $l_{i j}^{+}$and $q_{i j k}^{+}$terms calibrated.

Table 4. Two-equation ROM prediction errors.

\begin{tabular}{lccc}
\hline Reduced Order Model & $\chi_{0}$ & $\chi_{1}$ & $\chi_{2}$ \\
\hline uncalibrated & 2.122306 & 2.593450 & 16.011423 \\
$\nu_{T}$ calibration & 0.095617 & 0.113581 & 0.126317 \\
$l_{i j}^{+}$calibration & 0.065176 & 0.076025 & 0.090756 \\
$l_{i j}^{+}$and $q_{i j k}^{+}$calibration & 0.002539 & 0.001282 & 0.005296 \\
\hline
\end{tabular}

Fig. 9), where the amplitude is doubled after 120 seconds.

\section{Summary}

Reduced Order Models are crucial enablers of feedback flow control. The neglect of higher modes (truncation of the model) is one of main reasons of nonconformity of amplitudes, frequencies and phases of ROM and reference data from high-fidelity numerical simulations.

The calibration allows to create accurate least-dimensional models of the periodic flow around a bluff body, consisting of two or three degrees of freedom.

To find the optimal values of corrections: $l_{i j}^{+}$and $q_{i j k}^{+}$, in-house Genetic Algorithm with real-number gene encoding and combined truncation - roulette wheel selection is used. Fitness functions are related to the prediction errors of the flow models. The initial population is obtained by Monte-Carlo procedure (drawing the values of genes).

It has been shown, that presented calibration procedure leads to a great improvement of the quality of Reduced Order Models. For three-dimensional models, the agreement in phase and amplitude between ROM and DNS data is achieved. Two-dimensional models are still unstable, but the change of amplitude is much smaller than in the case of uncalibrated model. For a shorter prediction times it is possible to design two-dimensional, calibrated Reduced Order Model almost indistinguishable from high-fidelity reference data.

The number of generations required to converge the optimized solution depends on the number of calibrated parameters (genes modified in each iteration). For a small number of modified genes (e.g. $\nu_{T}$ calibration in Tab. 1 and 
Tab. $3, l_{i j}^{+}$calibration in Tab. 3), even two-three generations of Genetic Algorithm with population of 30 individuals lead to the noticeable improvement of model's quality. In that cases, Monte-Carlo search with large enough number of attempts should give comparable results.

In the cases when the number of modified genes is larger, the optimized results might be found when the analyzed population is of the size of hundreds of individuals and enough number of iteration steps (generations) is performed. The performed experiments have shown, that for $q_{i j k}^{+}$calibration of Reduced Order Galerkin Models of a flow around a circular cylinder, the Genetic Algorithm usually requires less than 15 iterations to converge.

For different values of Genetic Algorithm's parameters and initial populations, various optimized solutions can be found (the best of them are presented in previous section), although the finding of global optimum is not guaranteed. Thus further improvements in the model quality might be still possible, using different initial population or more iteration steps.

The methods presented in this paper will be used to calibrate Reduced Order Models of a transition from fixed-point to limit-cycle dynamics and the flows with deforming boundaries in aeroelastic applications.

\section{Acknowledgements}

This work was supported by the Polish Ministry of Science under grant no. N N501 225437.

We thank Bernd R. Noack, Laurent Cordier (CNRS, Universitè de Poitiers) and Gilead Tadmor (Northeastern University, Boston) for very stimulating discussions.

\section{References}

[1] N. Aubry, P. Holmes, J. Lumley and E. Stone. The dynamics of coherent structures in the wall region of a turbulent boundary layer. J. Fluid Mech., 192:115173, 1988. Doi:10.1017/S0022112088001818.

[2] J.E. Baker. Adaptive selection methods for genetic algorithms. In Proceedings of the 1st International Conference on Genetic Algorithms and their Applications, pp. 100-111, Pittsburgh, PA, USA, July 24“26, 1985.

[3] G. Berkooz, P. Holmes and J.L. Lumley. The proper orthogonal decomposition in the analysis of turbulent flows. Ann. Rev. Fluid Mech., 25:539-575, 1993. Doi:10.1146/annurev.fl.25.010193.002543.

[4] R. Bourguet, M. Braza, A. Dervieux and A. Sévrain. Transition features in transonic flow around a naca0012 airfoil by Navier-Stokes and low-order modeling. In Proceedings of West-East High Speed Flow Field ECCOMAS Conference, Moscow, Russia, 2007.

[5] D.J. Cavicchio. Adaptive Search using Simulated Evolution. PhD thesis, The University of Michigan, Ann Arbor, MI, USA, 1970.

[6] C.A.C. Coello. An updated survey of GA-based multiobjective optimization techniques. ACM Computing Surveys, 32(2):109-143, 2000. Doi:10.1145/358923.358929. 
[7] L. Cordier, B. Abou El Majd and J. Favier. Calibration of POD Reduced-Order Models using Tikhonov regularization. Int. J. Numer. Meth. Fluids, 63(2):269296, 2009.

[8] M. Couplet, C. Basdevant and C. Sagaut. Calibrated reduced-order PODGalerkin system for fluid flow modelling. J. Comp. Phys., 207(1):192-220, 2005. Doi:10.1016/j.jcp.2005.01.008.

[9] K.A. De Jong. An analysis of the behavior of a class of genetic adaptive systems. PhD thesis, The University of Michigan, Ann Arbor, MI, USA, 1975.

[10] M. Dorigo, V. Maniezzo and A. Colorni. The ant system: Optimization by a colony of cooperating agents. IEEE Transactions on Systems, Man, and Cybernetics Part B: Cybernetics, 26(1):29-41, 1996. Doi:10.1109/3477.484436.

[11] R.C. Eberhart and J. Kennedy. A new optimizer using particle swarm theory. In Proceedings of the Sixth International Symposium on Micro Machine and Human Science, pp. 39-43, 1995. Doi:10.1109/MHS.1995.494215.

[12] L.J. Fogel, A.J. Owens and M.J. Walsh. Artificial Intelligence through Simulated Evolution. John Wiley \& Sons, New York, USA, 1966.

[13] A.S. Fraser. Simulation of genetic systems by automatic digital computers. Austral. J. Biological Science, 20:484-491, 1957.

[14] G. Galletti, C.H. Bruneau, L. Zannetti and A. Iollo. Low-order modelling of laminar flow regimes past a confined square cylinder. J. Fluid Mech., 503:161170, 2004. Doi:10.1017/S0022112004007906.

[15] J. Gerhard, M. Pastoor, R. King, B.R. Noack, A. Dillmann, M. Morzyński and G. Tadmor. Model-based control of vortex shedding using low-dimensional Galerkin models. In 33rd AIAA Fluids Conference and Exhibit, Orlando, Florida, U.S.A., June 23-26, 2003. Paper 2003-4262

[16] D.E. Goldberg and K. Deb. A comparative analysis of selection schemes used in Genetic Algorithms. In Proceedings of Foundations of Genetic Algorithms, pp. 69-93, 1991.

[17] D.E. Goldberg, K. Deb and Korb B. Messy genetic algorithms: motivation, analysis, and first results. Complex Systems, 3(5):493-530, 1989.

[18] J.H. Holland. Outline for a logical theory of adaptive systems. Journal of the ACM, 9(3):297-314, 1957. Doi:10.1145/321127.321128.

[19] A. Iollo, S. Lanteri and J.A. Désidèri. Stability properties of POD-Galerkin approximations for the compressible Navier"Stokes equations. Technical Report 3589, INRIA, 1998.

[20] J. Kennedy and R.C. Eberhart. Particle swarm optimization. In Proceedings of IEEE International Conference on Neural Networks, volume 4, pp. 1942-1948, Perth, WA, Australia, 1995. Doi:10.1109/ICNN.1995.488968.

[21] P. Korošec and J. Šilc. Real-parameter optimization using stigmergy. In Proceedings of the Second International Conference on Bioinspired Optimization Methods and their Application, BIOMA 2006, pp. 73-84, 2006.

[22] T. Lieu, C. Farhat and M. Lesoinne. Reduced-order fluid/structure modeling of a complete aircraft configuration. Computer methods in applied mechanics and engineering, 195:5730-5742, 2006. Doi:10.1016/j.cma.2005.08.026.

[23] B.R. Noack. Low-dimensional modelling - post-transient natural flow. In CISM Course on Reduced-Order Modelling for Flow Control, Udine, Italy, 2008. Available from Internet: http://vento.pi.tu-berlin.de/ts/noackbr/introduction.php. 
[24] B.R. Noack, K. Afanasiev, M. Morzyński, G. Tadmor and F. Thiele. A hierarchy of low-dimensional models for the transient and post-transient cylinder wake. $J$. Fluid Mech., 497:335-363, 2003. Doi:10.1017/S0022112003006694.

[25] B.R. Noack, P. Papas and P.A. Monkewitz. The need for a pressure-term representation in empirical Galerkin models of incompressible shear flows. J. Fluid Mech., 523:339-365, 2005. Doi:10.1017/S0022112004002149.

[26] M. Nowak and M. Morzyński. Bio-inspired, structural optimization method based on the principle of constant strain energy density. In 12th AIAA/ISSMO Multidisciplinary Analysis and Optimization Conference, Victoria, British Columbia, Canada, September 10 - 12, 2003. Paper 2008-5992

[27] B. Podvin. On the adequacy of the ten-dimensional model for the wall layer. Phys. Fluids, 13:210-224, 2001. Doi:10.1063/1.1328741.

[28] D. Rempfer. Kohärente Strukturen und Chaos beim laminar-turbulenten Grenzschichtumschlag (transl.: Coherent structures and chaos of the laminarturbulent boundary-layer transition). PhD thesis, Fakultät Verfahrenstechnik der Universität Stuttgart, 1991. (Part of this work has been published by Rempfer, D. and Fazle, F.H. (1994) in J. Fluid Mech. 260 \& 275)

[29] D. Rempfer. On low-dimensional Galerkin models for fluid flow. Theoret. Comput. Fluid Dynamics, 14:75-88, 2000. Doi:10.1007/s001620050131.

[30] R.S. Rosenberg. Simulation of genetic populations with biochemical properties. PhD thesis, The University of Michigan, Ann Arbor, MI, USA, 1967.

[31] Y. Rouizi, M. Girault, Y. Favennec and D. Petit. Model reduction by the Modal Identification Method in forced convection: Application to a heated flow over a backward-facing step. Int. J. Thermal Sciences, 49:1354-1368, 2010. Doi:10.1016/j.ijthermalsci.2010.02.011.

[32] L. Sirovich. Turbulence and the dynamics of coherent structures. Quart. Appl. Math., 45:561-590, 1987.

[33] W. Stankiewicz, M. Morzyński, B.R. Noack and G. Tadmor. Reduced order Galerkin models of flow around NACA-0012 airfoil. Math. Model. Anal., 13(1):113-122, 2008. Doi:10.3846/1392-6292.2008.13.113-122.

[34] G. Tadmor, J. Gonzalez, O. Lehmann, B.R. Noack, M. Morzyński and W. Stankiewicz. Shift modes and transient dynamics in low order, design oriented Galerkin models. In 45th AIAA Aerospace Sciences Meeting and Exhibit, Reno, Nevada, U.S.A., Jan. 8-11, 2007. Paper 2007-0111

[35] G. Venter and J. Sobieszczanski-Sobieski. Particle swarm optimization. AIAA Journal, 41(8):1583-1589, 2003. Doi:10.2514/2.2111.

[36] T. Weise. Global Optimization Algorithms - Theory and Application. SelfPublished, 2009. Available from Internet: http://www.it-weise.de. 